\title{
Linear Dynamically Varying Versus Jump Linear Systems
}

\author{
Stephan Bohacek and Edmond Jonckheere \\ Dept of Electrical Engineering- Systems \\ University of Southern California \\ Los Angeles, CA 90089-2563 \\ (213) $740-4457$ \\ jonckhee@eudoxus.usc.edu
}

\begin{abstract}
The connection between linear dynamically varying (LDV) systems and jump linear systems is explored. LDV systems have been shown to be useful in controlling systems with "complicated dynamics". Some systems with complicated dynamics, for example Axiom A systems, admit Markov partitions and can be described, up to finite resolution, by a Markov chain. In this case, the control system for these systems can be approximated as Markovian jump linear systems. It is shown that (i) jump linear controllers for arbitrarily fine partitions exist if and only if the LDV controller exists; (ii) jump linear controllers stabilize the dynamical system; (iii) jump linear controllers are approximations of the LDV controller.
\end{abstract}

\section{Introduction}

Linear dynamically varying (LDV) controllers have been introduced as a technique to control systems with complicated dynamics [3], [4], [5], [10]. However, many systems with complicated dynamics can be described by symbolic dynamics and Markov chains. Hence, instead of approximating such a nonlinear system with an LDV system, one can approximate it with a linear system with parameters that vary according to a Markov chain, i.e. a Markovian jump linear system. Markovian jump linear systems have been the focus of extensive research [9], [8]. The relationship between the LDV and jump linear approximations of nonlinear systems will be investigated. It will be shown that the LDV approximation is the limit of a sequence of jump linear systems. Thus an LDV controller exists only if and only if a sequence of jump linear controllers exists. Furthermore, an LDV controller can be approximated by computing a jump linear controller. It will also be shown that when computing the latter controller the partition need not be Markov. That is, even if the induced symbolic dynamics is not a Markov chain, the jump linear controller (which incorrectly assumes that the symbolic dynamics is Markovian) will approximate the LDV controller. This is an important feature, since it is usually difficult to determine whether a partition is Markovian.

This paper proceeds as follows: In the next section the nonlinear tracking problem for systems with complicated dynamics is presented along with LDV controllers that solve this tracking problem. In section 3 jump linear systems are introduced along with some standard results. Section 4 shows how under certain conditions the nonlinear tracking problem described in section 2 may be described as a jump linear control problem. However, it is shown that this approach has difficulties in that stability of the closed loop nonlinear system cannot be easily proved. Section 6 consists of the main results.

\section{Tracking Systems with Complicated Dynamics via LDV control}

Consider the following nonlinear control problem: find a $u \in l_{2}$ such that $\|\varphi(k)-\theta(k)\| \rightarrow 0$ where

$$
\begin{aligned}
& \varphi(k+1)=f(\varphi(k), u(k)) \\
& \theta(k+1)=f(\theta(k), 0)
\end{aligned}
$$

and $f: \mathbb{R}^{n} \times \mathbb{R}^{m} \rightarrow \mathbb{R}^{n}, f(\Theta, 0) \subseteq \Theta$ with $\Theta$ compact, $\theta(0), \varphi(0) \in \Theta$ and $f \in C^{1}$. This problem is especially interesting when $f$ displays complicated dynamics on $\Theta$, in particular, nontrivial recurrence. Define the set of recurrent points $R(f)$ and the set of periodic point $P(f)$. Complicated dynamics occurs when $P(f) \mp R(f)$, or simply if $P(f)$ is not a stable attractor. Such systems have been extensively study by Birkhoff, Moser, Halmos, Smale, Kolmogorov, Arnold, Sinai, etc.

The tracking error is defined as $x(k)=\varphi(k)-\theta(k)$. Then system 1 reduces to

$$
\begin{aligned}
x(k+1)= & A_{\theta(k)} x(k)+B_{\theta(k)} u(k) \\
& +\eta(\theta(k), x(k), u(k)) \\
\theta(k+1)= & f(\theta(k), 0)
\end{aligned}
$$

where $A_{\theta}=\frac{\partial f}{\partial x}(\theta, 0), B_{\theta}=\frac{\partial f}{\partial u}(\theta, 0)$ and $\eta$ accounts for nonlinear terms. It is not hard to show that if $x$ and $u$ are small, then system 2 can be approximated as a 
linear dynamically varying (LDV) system:

$$
\begin{aligned}
& x(k+1)=A_{\theta(k)} x(k)+B_{\theta(k)} u(k) \\
& \theta(k+1)=f(\theta(k), 0) .
\end{aligned}
$$

These LDV systems have been extensively studied in [3], [4], [5] and [10]. Only a brief introduction will follow. An LDV system is defined as

$$
\begin{aligned}
x(k+1) & =A_{\theta(k)}^{L D V} x(k)+B_{\theta(k)}^{L D V} u(k) \\
z(k) & =\left[\begin{array}{c}
C_{\theta(k)}^{L D V} x(k) \\
D_{\theta(k)}^{L D V} u(k)
\end{array}\right] \\
\theta(k+1) & =f(\theta(k), 0) .
\end{aligned}
$$

with $A^{L D V}: \Theta \rightarrow \mathbb{R}^{n \times n}, B^{L D V}: \Theta \rightarrow \mathbb{R}^{n \times m}, C^{L D V}$ : $\Theta \rightarrow \mathbb{R}^{p \times n}, D^{L D V}: \Theta \rightarrow \mathbb{R}^{q \times m}$ and $f: \Theta \rightarrow \Theta$, with $\Theta$ compact. If the maps $A, B, C, D \in C^{0}$, then system 3 is a continuous LDV.

We say that the pair $\left(A^{L D V}, f\right)$ is exponentially stable if system 3 is exponentially stable. That is, for $u=0$ and $\theta_{o} \in \Theta$ there exist an $\alpha\left(\theta_{o}\right)<1$ and a $\beta\left(\theta_{o}\right)<\infty$ such that if $\theta(0)=\theta_{o}$, then $\|x(k)\|<$ $\beta\left(\theta_{0}\right) \alpha\left(\theta_{0}\right)^{k}\|x(0)\|$. Similarly, the pair $\left(A^{L D V}, f\right)$ is uniformly exponentially stable if the pair $\left(A^{L D V}, f\right)$ is exponentially stable and $\alpha$ and $\beta$ can be chosen independent of $\theta(0)$. The triple $\left(A^{L D V}, B^{L D V}, f\right)$ is stabilizable if there exists a bounded feedback $F: \Theta \rightarrow$ $\mathbb{R}^{m \times n}$ such that $\left(A^{L D V}+B^{L D V} F, f\right)$ is exponentially stable. Note that uniform exponential stability is not required for a system to be stabilizable. The triple $\left(A^{L D V}, C^{L D V}, f\right)$ is uniformly detectable if there is a uniformly bounded map $H: \Theta \rightarrow \mathbb{R}^{n \times p}$ such that $\left(A^{L D V}+H C^{L D V}, f\right)$ is uniformly exponentially stable.

It was shown in [2] and [3] that if the LDV system 3 induced by $f$ is uniformly exponentially stabilized by the control $u(k)=F_{\theta(k)} x(k)$, then the nonlinear system 2 , with control $u(k)=F_{\theta(k)} x(k)$, is locally uniformly exponentially stable. By definition locally uniformly exponentially stable means that there exist $\alpha<1, \beta<\infty$ and $\gamma>0$ such that if $\|x(0)\|=\|\varphi(0)-\theta(0)\|<\gamma$ then $\|x(k)\|<\beta \alpha^{k}\|x(0)\|$ where $\alpha, \beta$ and $\gamma$ can be taken independent of the initial condition $\theta_{o}$, i.e. uniformly in $\theta_{o}$ and locally in $x$. Therefore, we say that the dynamical system $f$ is LDV stabilizable if the LDV system induced by $f$ is stabilizable.

One of the main results from [3] is:

Theorem 1 Suppose 3 is a continuous, uniformly detectable $L D V$ system with $D_{\theta}^{L D V '} D_{\theta}^{L D V}>0$ for $\theta \in$ $\Theta$. Then system 3 is LDV stabilizable if and only if there exists a bounded function $X: \Theta \rightarrow \mathbb{R}^{n \times n}$ with $X_{\theta}^{\prime}=X_{\theta} \geq 0$ that satisfies the functional discrete time algebraic Riccati equation

$$
\begin{array}{r}
X_{\theta}=A_{\theta}^{L D V^{\prime}} X_{f(\theta)} A_{\theta}^{L D V}+C_{\theta}^{L D V^{\prime}} C_{\theta}^{L D V} \\
-A_{\theta}^{L D V^{\prime}} X_{f(\theta)} B_{\theta}^{L D V}
\end{array}
$$

$$
\begin{array}{r}
\times\left(D_{\theta}^{L D V '} D_{\theta}^{L D V}+B_{\theta}^{L D V \prime} X_{f(\theta)} B_{\theta}^{L D V}\right)^{-1} \\
\times B_{\theta}^{L D V '} X_{f(\theta)} A_{\theta}^{L D V}
\end{array}
$$

In this case the control

$$
\begin{array}{r}
u^{L D V}(k)=F_{\theta(k)}^{L D V} x(k) \\
=-\left(D_{\theta(k)}^{L D V^{\prime}} D_{\theta(k)}^{L D V}+B_{\theta(k)}^{L D V^{\prime}} X_{f(\theta(k))} B_{\theta(k)}^{L D V}\right)^{-1} \\
\times B_{\theta(k)}^{L D V^{\prime}} X_{f(\theta(k))} A_{\theta(k)}^{L D V}
\end{array}
$$

is optimal in the sense that it minimizes the quadratic cost

$$
\begin{aligned}
V\left(\theta_{o}, u, x_{o}\right)= & \sum_{k=0}^{\infty} x(k)^{\prime} C_{f^{k}\left(\theta_{o}\right)}^{L D V^{\prime}} C_{f^{k}\left(\theta_{o}\right)}^{L D V} x(k) \\
& +u(k)^{\prime} D_{f^{k}\left(\theta_{o}\right)}^{L D V^{\prime}} D_{f^{k}\left(\theta_{o}\right)}^{L D V} u(k) .
\end{aligned}
$$

Furthermore, this control uniformly exponentially stabilizes the system and $x_{o}^{\prime} X_{\theta_{o}} x_{o}=\min _{u} V\left(\theta_{o}, u, x_{o}\right)$ and $X$ is a continuous function.

Under some mild assumptions on the dynamical system $f$, it is known that $f$ has many structural properties. These properties can be used to determine approximate solutions to 4 . In [3] techniques based on a dense set periodic points, a dense orbit and recurrence are developed. Another technique based on a probabilistic interpretation of $f$ is developed here.

\section{Jump Linear Systems}

A jump linear system is defined as follows:

$$
\begin{aligned}
x(k+1) & =A_{s(k)}^{J L} x(k)+B_{s(k)}^{J L} u(k) \\
z(k) & =\left[\begin{array}{c}
C_{s(k)}^{J L} x(k) \\
D_{s(k)}^{J L} u(k)
\end{array}\right]
\end{aligned}
$$

with $s(k)$ a Markov chain that takes values in a finite set $\{1,2, \cdots, M\}$ with transition probabilities

$$
P\left(s(k+1)=j \mid s(k)=i, s(k-1)=l_{1}, \cdots\right)=p_{i, j} .
$$

Thus the parameters $A^{J L}, B^{J L}, C^{J L}, D^{J L}$ are matrix valued Markov chains. At time $k$ it is assumed that only $s(k)$ and $x(k)$ are known.

System 6 is stochastically stabilizable if there exists a function $F^{J L}:\{1,2 \cdots M\} \rightarrow \mathbb{R}^{m \times n}$ such that the closed loop jump linear system

$$
x(k+1)=\left(A_{s(k)}^{J L}+B_{s(k)}^{J L} F_{s(k)}^{J L}\right) x(k)
$$

is stochastically stable, where stochastically stable means that there exist $\alpha<1$ and $\beta<\infty$ such that for $1 \leq i \leq M$,

$$
E(\|x(k)\| \mid s(0)=i)<\beta \alpha^{k}\|x(0)\| .
$$

Similarly, system 6 is stochastically detectable if there exists a function $H^{J L}:\{1,2 \cdots M\} \rightarrow \mathbb{R}^{n \times p}$ such that 
the closed loop jump linear system

$$
x(k+1)=\left(A_{s(k)}^{J L}+H_{s(k)}^{J L} C_{s(k)}^{J L}\right) x(k)
$$

is stochastically stable.

As shown in [9], [8], assuming that $\left(D_{s(k)}^{J L^{\prime}} D_{s(k)}^{J L}\right)$ is invertible, the optimal linear quadratic controller for these stochastic systems is characterized by the existence of a function $Y:\{1,2, \cdots M\} \rightarrow \mathbb{R}^{n \times n}$ with $Y_{i}^{\prime}=Y_{i} \geq 0$ such that:

$$
\begin{aligned}
& Y_{s(k)}=A_{s(k)}^{J L \prime} \hat{Y}_{s(k+1) \mid s(k)} A_{s(k)}^{J L}+C_{s(k)}^{J L \prime} C_{s(k)}^{J L} \\
& -A_{s(k)}^{J L \prime} \hat{Y}_{s(k+1) \mid s(k)} B_{s(k)}^{J L} \\
& \times\left(D_{s(k)}^{J L \prime} D_{s(k)}^{J L}+B_{s(k)}^{J L \prime} \hat{Y}_{s(k+1) \mid s(k)} B_{s(k)}^{J L}\right)^{-1} \\
& \times B_{s(k)}^{J L \prime} \hat{Y}_{s(k+1) \mid s(k)} A_{s(k)}^{J L}
\end{aligned}
$$

where

$$
\hat{Y}_{s(k+1) \mid s(k)}=E\left(Y_{s(k+1)} \mid s(k)\right)=\sum_{j=1}^{M} p_{s(k), j} Y_{j} .
$$

Equation 7 defines a system of coupled Riccati equations. If a such a function exists, then a control is

$$
\begin{aligned}
u(k)= & F_{s(k)}^{J L} x(k) \\
= & -\left(D_{s(k)}^{J L^{\prime}} D_{s(k)}^{J L}+B_{s(k)}^{J L \prime} \hat{Y}_{s(k+1) \mid s(k)} B_{s(k)}^{J L}\right)^{-1} \\
& \times B_{s(k)}^{J L \prime} \hat{Y}_{s(k+1) \mid s(k)} A_{s(k)}^{J L} x(k) .
\end{aligned}
$$

This control is optimal in the sense that

$$
\begin{aligned}
u= & \arg \min _{u \in U_{J L}} E\left(\sum_{k=1}^{\infty} x(k)^{\prime} C_{s(k)}^{J L^{\prime}} C_{s(k)}^{J L} x(k)\right. \\
& \left.+u(k)^{\prime} D_{s(k)}^{J L^{\prime}} D_{s(k)}^{J L} u(k)\right)
\end{aligned}
$$

where $U_{J L}$ is the set of $u$ such that $u(k)$ depends only on $x(l)$ and $s(l)$ for $l \leq k$. That is, $u \in U_{J L}$ implies that $u(k) \in \mathcal{F}_{k}$ where $\mathcal{F}_{k}$ is the sigma algebra generated by $s(l), l \leq k$ and $u(k) \in \mathcal{F}_{k}$ denotes that $u(k)$ is $\mathcal{F}_{k}$ measurable.

If a solution to 7 exists and system 6 is stochastically detectable, then the control 8 is stochastically stabilizing. Furthermore, a stochastically stabilizing controller exists if and only if a positive semi-definite solution to 7 exists.

Techniques to solve 7 are discussed in [1] and [6].

\section{LDV System Inducing Jump Linear System}

\subsection{Dynamical System Inducing a Markov Chain}

A dynamical system may admit a Markov chain as follows:

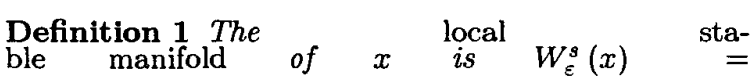

$\left\{y:\left\|f^{k}(x)-f^{k}(y)\right\| \stackrel{k \rightarrow \infty}{\rightarrow} 0\right.$ and $\left.\|x-y\|<\varepsilon\right\}$ Likewise, the local unstable manifold of $x$ is $W_{\varepsilon}^{s}(x)=$ $\left\{y:\left\|f^{-k}(x)-f^{-k}(y)\right\| \stackrel{k \rightarrow \infty}{\rightarrow} 0\right.$ and $\left.\|x-y\|<\varepsilon\right\}$.

Definition $2 A$ subset $R \subset \Theta$ is a rectangle if $\operatorname{diam}(R)<\delta$ and $W_{\varepsilon}^{s}(x) \cap W_{\varepsilon}^{u}(y) \subset R$ for every $x, y \in R$, where $\delta$ and $\varepsilon$ are small enough and depend on the system (see [12] for details). A rectangle is proper if $R=\operatorname{cl}(\operatorname{int}(R))$.

Definition $3 A$ family of proper rectangles $\mathcal{R}=$ $\left\{R_{1}, R_{2}, \cdots R_{M}\right\}$ is a Markov partition if

1. $\bigcup_{i=1}^{N} R_{i}=\Theta$

2. $R_{i} \cap R_{j}=\partial R_{i} \cap \partial R_{j}$ for $i \neq j$.

3. For every $1 \leq i, j \leq M$ such that $f\left(R_{i}\right) \operatorname{nint}\left(R_{j}\right) \not \emptyset$ and every $x \in R_{i} \cap$ $f^{-1}\left(\operatorname{int}\left(R_{j}\right)\right)$ we have

$$
f\left(W_{\varepsilon}^{s}(x) \cap R_{i}\right) \subset W_{\varepsilon}^{s}(f(x)) \cap R_{j} .
$$

4. For every $1 \leq i, j \leq M$ such that $f^{-1}\left(R_{i}\right) \operatorname{nint}\left(R_{j}\right) \neq \emptyset$ and every $x \in R_{i} \cap$ $f\left(\operatorname{int}\left(R_{j}\right)\right)$ we have

$$
f^{-1}\left(W_{\varepsilon}^{u}(x) \cap R_{i}\right) \subset W_{\varepsilon}^{u}\left(f^{-1}(x)\right) \cap R_{j} .
$$

Once a Markov partition has been chosen, then there exist a matrix $T=\left\{t_{i, j}\right]$ with $t_{i, j} \in\{0,1\}$, a subset of allowable sequences of $M$ symbols

$$
\Sigma_{T}=\left\{s: \mathbb{Z} \rightarrow\{1,2, \cdots M\}: t_{s(k), s(k+1)}=1, \forall k\right\}
$$

and a continuous map

$$
\begin{aligned}
h & : \quad \Sigma_{T} \rightarrow \Theta \\
h(s) & =\bigcap_{k=-\infty}^{\infty} f^{-k}\left(R_{s(k)}\right)
\end{aligned}
$$

which implies that

$$
h(\{s: s(0)=i\})=R_{i} .
$$

Furthermore, $h$ is such that the diagram

$$
\begin{array}{lll}
\Sigma_{T} & \stackrel{\sigma}{\rightarrow} & \Sigma_{T} \\
\downarrow h & & \downarrow h \\
\Theta & \stackrel{f}{\rightarrow} & \Theta
\end{array}
$$

commutes, where $\sigma$ is the shift operator defined by $\sigma(s)(k)=s(k+1)$. Hence, $f$ is semi-conjugate to the topological Markov chain $\left(\sigma, \Sigma_{T}\right)$. Furthermore, there exists a $\sigma$ invariant measure on $\Sigma_{T}$ such that $s(k)$ is a Markov chain with

$$
P(s(k+1)=j \mid s(k)=i)=p_{i, j}
$$

and $h$ is a measure preserving map, i.e. $P\left(s \in h^{-1}(E)\right)=\mu(\theta \in E)$ where $\mu$ is an invariant measure for $f$. Thus the dynamics of $f$ is described by a Markov chain. 
Bowen [7] showed that systems that satisfy Axiom A have Markov partitions with arbitrarily small diameter of the rectangles. Bowen's result can be extended to nonuniform hyperbolic systems [11] and to systems that satisfy a local product structure [13].

\subsection{LDV System Inducing a Jump Linear Sys- tem}

We now show how a LDV system may give rise to a jump linear system. As described above, depending on the dynamical system $f$, there may exist a Markov partition and the dynamics of $f$ can be described by a Markov chain $s(k)$ on the finite set of symbols $\{1,2, \cdots M\}$ with transition probabilities $p_{i, j}$. This leads to a jump linear system as follows: For each cell $R_{i}$ of the Markov partition $\mathcal{R}=\left\{R_{1}, R_{2}, \cdots R_{M}\right\}$ define a point $\phi_{i} \in \operatorname{int}\left(R_{i}\right)$ for $1 \leq i \leq M$. Set

$$
\begin{aligned}
& A_{s(k)}^{J L}=A_{\phi_{s(k)}}^{L D V}=\frac{\partial f}{\partial x}\left(\phi_{s(k)}, 0\right) \\
& B_{s(k)}^{J L}=B_{\phi_{s(k)}}^{L D V}=\frac{\partial f}{\partial u}\left(\phi_{s(k)}, 0\right) .
\end{aligned}
$$

Then $A_{s(k)}^{J L}$ is a Markov chain which takes values in $\left\{A_{\phi_{1}}^{L D V}, A_{\phi_{2}}^{L D V}, \cdots A_{\phi_{M}}^{L D V}\right\}$ and $B_{s(k)}^{J L}$ is a Markov chain which takes values in $\left\{B_{\phi_{1}}^{L D V}, B_{\phi_{2}}^{L D V}, \cdots B_{\phi_{M}}^{L D V}\right\}$. Thus we have the jump linear system:

$$
x^{J L}(k+1)=A_{s(k)}^{J L} x(k)+B_{s(k)}^{J L} u(k)
$$

Note that if $\max _{i}\left(\operatorname{diam}\left(R_{i}\right)\right)$ is small and $h(s)=$ $\theta(0)$, then $A_{s(k)}^{J L} \approx A_{\theta(k)}^{L D V}$ and $B_{s(k)}^{J L} \approx B_{\theta(k)}^{L D V}$, and therefore, $x^{J L}(k) \approx x^{L D V}(k)$. Hence, the jump linear system approximates the LDV system. The smaller the size of the cells $R_{i}$ the better the approximation and as $\max _{i}\left(\operatorname{diam}\left(R_{i}\right)\right) \rightarrow 0$, and fixed $k$, we have $x^{J L}(k) \rightarrow x^{L D V}(k)$.

\section{Jump Linear Control of Complicated Dynamics}

From the above, it is clear that some dynamical systems induce jump linear systems. Since there has been extensive work on jump linear systems, it seems feasible to stabilize system 2 with a jump linear controller. However, it will now be shown that such an approach is more difficult than it appears.

Suppose $f$ induces Markov partitions with arbitrarily small rectangles. Then for any of these partitions one can construct a jump linear controller. Applying this controller to system 2 yields:

$$
\begin{aligned}
x(k+1)= & \left(A_{s(k)}^{J L}+B_{s(k)}^{J L} F_{s(k)}^{J L}\right) x(k) \\
& +\eta\left(\theta(k), x(k), F_{s(k)}^{J L} x(k)\right)
\end{aligned}
$$

where, if the partition is fine enough, i.e. $\max \left(\operatorname{mesh}\left(R_{i}\right)\right)$ is small enough, $A_{s(k)}^{J L} \approx$ $\frac{\partial f}{\partial x}\left(h\left(\sigma^{k}(s)\right)\right)=\frac{\partial f}{\partial x}(\theta(k))$.

Since $f \in C^{1}$ it is not hard to show that if $x(k)$ is small, then $\eta\left(\theta(k), x(k), F_{s(k)}^{J L} x(k)\right)$ is small. Furthermore, it is true that if $\eta\left(\theta(k), x(k), F_{s(k)}^{J L} x(k)\right)$ is small, the system 10 is stochastically stable. In this case, if $\|x(0)\|$ is small, $E(\|x(k)\|)$ is small for all $k$. However, there may be a non-zero probability that $\|x(k)\|$ is not small. Thus, there may be a non-zero probability that $\eta\left(\theta(k), x(k), F_{s(k)}^{J L} x(k)\right)$ is not small. This may imply that there is a non-zero probability that the system is unstable, hence $E\|x(k)\| \rightarrow \infty$. Of course, using a Chebyshev type inequality one can show that by limiting $\|x(0)\|$ the closed loop system, with nonlinear perturbation, is stable with a probability close to one.

Furthermore, suppose the desired trajectory $\{\theta(k)\}$ is a fixed point, and the probability of staying in the cell containing the fixed point is not one. In this case, the probability of staying in the cell containing the fixed point for all time is zero. Therefore, stochastic stability does not directly imply that the jump linear system is stable at the fixed point. The difficulty is that stochastic stability implies stability over the average orbit $\{\theta(k)\}$. When a particular orbit is chosen, stochastic stability cannot guarantee anything about the stability along this orbit.

\section{Main Results}

Next it will be shown that if the nonlinear system is LDV stabilizable, then for a fine enough partition, the jump linear system stabilizes the nonlinear system (proposition 2). Conversely, if as the partition is refined, the solution to the jump linear coupled Riccati equations 7 remains bounded, then the system is LDV stabilizable (proposition 4). In this case, as the partition is refined, the jump linear controller approaches the LDV controller (theorem 6). Moreover, this process is robust to errors in the Markov partition. That is, if the partition is incorrectly assumed to be Markov, the resulting jump linear controller still approximates the LDV controller and, if the partition is fine enough, stabilizes the nonlinear system.

As discussed in section 4.2, a Markov partition and an LDV system induce a Markovian jump linear system. Here the requirement that the partition be Markov is dropped. Instead, given a partition, a jump linear system is defined in the same way as in section 4.2 , with transition probabilities defined via $p_{i, j}:=$ $P(s(k+1)=j \mid s(k)=i)$. Since the jump linear system assumes that the partition is Markovian, there will be some error. However, if the partition is fine enough, i.e. $\operatorname{mesh}(\mathcal{R})$ is small enough, then the error in assuming the partition is Markov is small. In this way 
an LDV system and a partition induce a jump linear system.

Proposition 2 Assume that the map $f$ is LDV stabilizable. In this case there exists a $\delta>0$ such that if mesh $(\mathcal{R})<\delta$, then the jump linear system induced by $f$ and $\mathcal{R}$ is stochastically stabilizable. Furthermore, assuming that $C^{L D V}: \Theta \rightarrow \mathbb{R}^{p \times n}$ and $D^{L D V}: \Theta \rightarrow \mathbb{R}^{q \times m}$ are continuous, if $\operatorname{mesh}(\mathcal{R})<\delta$, then there is a bound $\bar{Y}$ on $Y$ the solution to the coupled Riccati equations that is independent of the partition.

The following dual result can be proved in the same fashion as the above proposition.

Corollary 3 Assume that $C^{L D V}: \Theta \rightarrow \mathbb{R}^{p \times n}$ is continuous and $\left(A^{L D V}, C^{L D V}, f\right)$ is $L D V$ detectable. There exists a $\delta>0$ such that if mesh $(\mathcal{R})<\delta$, then the jump linear system induced by $A^{L D V}, C^{L D V}$, $f$ and $\mathcal{R}$ is stochastically detectable. Furthermore, for mesh $(\mathcal{R})<\delta$, the $\alpha_{d}$ and $\beta_{d}$ in the definition of stochastic detectability can be taken independent of the partition.

Let $\left\{\mathcal{R}^{t}\right\}$ be a sequence of partitions of $\Theta$. Then each partition induces a solution $Y^{t}$ to the coupled Riccati equations 7 and a jump linear controller $F^{J L, t}$.

Proposition 4 Assume that $C^{L D V}: \Theta \rightarrow \mathbb{R}^{p \times n}$, $D^{L D V}: \Theta \rightarrow \mathbb{R}^{q \times m}$ are continuous, $\left(A^{L D V}, C^{L D V}, f\right)^{\prime}$ is LDV uniformly detectable and there exists a sequence of partitions $\left\{\mathcal{R}^{t}\right\}$ with mesh $\left(\mathcal{R}^{t}\right) \rightarrow 0$ such that $\left\|Y_{i}^{t}\right\|<\bar{Y}$ for all $i$. In this case, the $L D V$ induced by $f$ is stabilizable.

Combining propositions 2 and 4 yields:

Theorem 5 Let $C^{L D V}: \Theta \rightarrow \mathbb{R}^{p \times n}$ and $D^{L D V}: \Theta \rightarrow$ $\mathbb{R}^{q \times m}$ be continuous with $D_{\theta}^{L} D V^{\prime} D_{\theta}^{L D V}>0$, and let $\left(A^{L D V}, C^{L D V}, f\right)$ be uniformly detectable. The $L D V$ induced by $f$ is stabilizable if and only if for any sequence of partitions $\mathcal{R}^{t}$ such that mesh $\left(\mathcal{R}^{t}\right) \rightarrow 0$ the Markov jump linear systems induced by $f$ and $\mathcal{R}^{t}$ are stabilizable with bounded optimal quadratic cost, where the bound does not depend on $t$.

Thus the existence of a stabilizing LDV controller is linked to the existence of a series of stabilizing jump linear controllers. Now we show that actually these controllers are nearly identical.

Theorem 6 Let $C^{L D V}: \Theta \rightarrow \mathbb{R}^{p \times n}$ and $D^{L D V}$ : $\Theta \rightarrow \mathbb{R}^{q \times n}$ be continuous with $\vec{D}_{\theta}^{L D V I} D_{\theta}^{L D V}>0$, and let $\left(A^{L D V}, C^{L D V}, f\right)$ be uniformly detectable. Assume that $f$ is $L D V$ stabilizable or equivalently assume that there exists a bounded sequence of solutions $Y^{t}$ to the coupled Riccati equations 7 associated with a sequence of partitions with mesh $\left(\mathcal{R}^{t}\right) \rightarrow 0$; then

$$
\sup _{\theta \in \Theta}\left\|X_{\theta}-Y_{g(\theta)}^{t}\right\| \rightarrow 0 \text { as } t \rightarrow \infty
$$

where $X$ solves the functional Riccati equation and $Y^{t}$ solve the coupled Riccati equations.

\section{References}

[1] H. Abou-Kandil, G. Freiling, and G. Jank. Solution and asymptotic behavior of coupled riccati equations in jump linear systems. IEEE transactions on automatic control, 39:1631-1636, 1994.

[2] S. Bohacek and E. Jonckheere. Linear dynamically varying $\mathrm{H}$-infinity control of complicated dynamics. Submitted to SIAM J. of Control and Optimization.

[3] S. Bohacek and E. Jonckheere. Linear dynamically varying $\mathrm{LQ}$ control of systems with complicated dynamics. Submitted to IEEE Transactions on Automatic Control.

[4] S. Bohacek and E. Jonckheere. Linear dynamically varying $\mathrm{H}$-infinity control chaos. In Nonlinear Control Systems Design Symposium, pages 744-749. IFAC, 1998.

[5] S. Bohacek and E. Jonckheere. Structural stability of LDV controllers. In $C D C, 1998$.

[6] I. Borno and Z. Gajic. Parallel algorithm for solving coupled algebraic Lyapunov equations of discretetime jump linear systems. Computers Mathematics and Applications, 30:1-4, 1995.

[7] R. Bowen. Markov partitions for axiom A differmorphisms. American Journal of Mathematics, 92:725$747,1970$.

[8] O. Costa and M. Fragoso. Discrete-time LQoptimal control problems for infinite Markov parameter system. IEEE Transactions on Automatic Control, 40(12):2076-2088, 1995.

[9] Y. Ji and H. Chizeck. Jump linear quadratic Gaussian control: Steady-state solution and testable conditions. Control-Theory and Advanced Technology, 10(3):1459-1474, 1990.

[10] E. A. Jonckheere and S. K. Bohacek. Ergodic and topological aspects of linear dynamically varying (LDV) control. MTNS98, 1998.

[11] A. Katok and B. Hasselblatt. Introduction to the Modern Theory of Dynamical Systems. Cambridge University Press, 1995.

[12] R. Mane. Ergodic Theory and Differentiable Dynamics. Springer-Verlag, 1980.

[13] D. Ruelle. Thermodynamic formalism, volume 5 of Encyclopedia of mathematics and its applications. Addison-Wesley, 1978. 